\title{
An exploratory study of two-dimensional shear-wave elastography in the diagnosis of acute compartment syndrome
}

Jun Zhang ${ }^{1}$, Wanfu Zhang ${ }^{2}$, Huihui Zhou', Lin Sang ${ }^{1}$, Lina Liu', Yuanyuan Sun ${ }^{1}$, Xue Gong ${ }^{1}$, Hao Guan² and Ming $Y u^{1^{*}}$ (D)

\begin{abstract}
Background: Two-dimensional shear-wave elastography (2D-SWE) is an ultrasound elastography technique that uses shear waves to quantitatively measure tissue stiffness and it has recently been developed as a safe, real-time, and noninvasive imaging technique. The purpose of this study was to investigate the value of 2D-SWE in the diagnosis and treatment of acute compartment syndrome (ACS).
\end{abstract}

Methods: 2D-SWE was used to measure the elasticity values of the main muscles in the superficial compartments of the calf in 212 healthy volunteers, and the difference in the muscle elasticity values between different gender and age groups were analyzed. Nine patients with clinical suspicion of ACS were included in this study and 2D-SWE was used to measure the elasticity values of the muscles on the affected and unaffected sides, and a comparative analysis was performed.

Results: The mean elasticity values of the tibialis anterior (TA), peroneus longus (PL), and gastrocnemius medialis (GA) muscles in the relaxed state of the 212 healthy volunteers were $25.4 \pm 3.2 \mathrm{kPa}, 15.7 \pm 1.5 \mathrm{kPa}$, and $12.1 \pm 2.1 \mathrm{kPa}$, respectively. No statistically significant differences was observed in the elasticity values of the same muscle under the state of relaxation in different gender and age groups $(p>0.05)$. A statistically significant difference in the elasticity values of the muscle between the affected and unaffected sides in the fasciotomy group $(p<0.05, n=5)$ was observed. In contrast, no difference in the elasticity values of the muscle between the affected and unaffected sides in the conservative group $(p>0.05, n=4)$ was observed. There was a statistically significant difference in the elasticity values of the muscle on the affected side in the two treatment groups $(p<0.05)$.

Conclusions: When the ACS occurs, the muscle elasticity of the affected limb increases significantly. 2D-SWE is expected to be a new noninvasive technique for the assessment of ACS and may provide a potential basis for clinical diagnosis and treatment.

Keyword: Ultrasound 2D shear-wave elastography, Acute compartment syndrome, Elasticity value of muscle, Noninvasive measurement

*Correspondence: guanhao@hotmail.com; yumingfmmu@126.com ${ }^{1}$ The Department of Ultrasound, Xijing Hospital, The Fourth Military Medical University, No. 127 Changle West Rd, Xi'an 710032, Shaanxi, China ${ }^{2}$ The Department of Burns and Cutaneous Surgery, Xijing Hospital, The Fourth Military Medical University, No. 127 Changle West Rd, Xi'an 710032, Shaanxi, China

\section{Background}

Acute compartment syndrome (ACS) is a clinical surgical emergency that refers to an increase in intra-compartmental pressure (ICP) caused by various reasons, such as fractures, crush injuries, and thermal injuries, resulting in a decrease in perfusion pressure, leading to 
hypoxemia of the tissues [1]. Decreased tissue perfusion can lead to irreversible necrosis that may result in limb dysfunction, amputation, and even death. The forearm and the lower leg, especially the latter, are more prone to develop compartment syndrome owing to their doublebone structure, and thick, tough, and inelastic interosseous membrane and fascia [2]. The lower leg is the most common location of ACS, which consists of the following four compartments: anterior, lateral, superficial posterior, and deep posterior, with the anterior and lateral compartments most frequently affected, especially the anterior compartment. The tibialis anterior (TA), peroneus longus (PL), and gastrocnemius (GA) are the main muscles in the first three compartments, respectively [3]. Currently, the diagnosis of ACS mainly depends on the clinical manifestations and measurement of the ICP by some invasive methods, such as the Whitesides needle manometer, a slit or wick catheter [4]. However, the judgment of clinical manifestations is subjective and delayed. To date, no definite pressure threshold value that can be used as an indication of early incision decompression is available [5]. Moreover, the measurement methods of ICP are invasive, increasing the risk of infection and patient's symptoms [6-8]. Therefore, we wish to find a real-time, noninvasive, safe, and accurate assessment method for the diagnosis and treatment of ACS.

Ultrasonography, one of several main clinical imaging techniques, has distinct strengths for the evaluation of superficial soft-tissue diseases. Two-dimensional shear-wave elastography (2D-SWE) is an ultrasound elastography technique that uses shear waves to quantitatively measure tissue stiffness, and it has recently been developed as a safe, real-time, and noninvasive imaging technique [9]. By quantifying mechanical and elastic tissue properties, SWE complements the diagnosis information obtained via gray-scale US and color Doppler US. As a transverse wave, the shear wave occurs in an elastic medium that is subject to a periodic shear force, and shear is defined as change in the shape of a substance layer without volume change, produced by a pair of equal forces working in opposite directions along the two opposed sides of the layer [10]. To characterize tissue stiffness with SWE does not require knowledge of applied stress [11, 12].
Currently, 2D-SWE is relatively mature and widely used in the examination and evaluation of the superficial organs (such as thyroid, breast, and skin, among others) [13-15] and abdominal organs (such as liver, spleen, and kidney, among others) [16-18]. The application and efficacy of 2D-SWE in the evaluation of several traumatic and pathological conditions of various musculoskeletal soft tissues, including the muscles [19-22], tendons [23, 24], ligaments [25], and nerves [26-28] have been reported. Quantitative elastography can not only greatly help by providing a parameter that can influence the diagnosis, but also providing a way to monitor the effectiveness of a treatment by quantifying the changes in the mechanical state of the muscle [28]. The ICP was increasing when ACS occurred, and Steinberg observed a strong correlation between the stiffness of the muscle compartment and the ICP [29]. The measurement of the elasticity value of muscle tissue has been suggested as one of the most promising techniques to detect and objectify an ACS [30]. However, the utility of 2D-SWE for diagnosis of ACS is still unclear, mainly owning to the lack of standardization of the elasticity values of muscles in healthy subjects.

The aim of our study was to evaluate the muscles in the fascia compartment of healthy volunteers and suspectedACS patients by multimodal ultrasound (two-dimensional, color Doppler, and 2D-SWE), and to explore whether multimodal ultrasound (especially 2D-SWE) could be used as an effective inspection method for the diagnosis and treatment of ACS.

\section{Methods}

\section{Research participants}

We performed a prospective analysis of nine patients with limb swelling suspected of acute compartment syndrome and 212 healthy volunteers who were included in this study with their consent (Tables 1, 2, 3). The inclusion criteria of the volunteers were as follows: age range: 20-70 years, body mass index (BMI) in the normal range (18.5-23.9, Chinese reference standard for BMI), and no history of musculoskeletal system diseases or trauma or neurological diseases. The volunteers were divided into three groups according to the World Health Organization (WHO) classification: group I, young people

Table 1 Mean elasticity values for TA, PL, GA in the gender groups ( $n=212$, mean \pm standard deviation)

\begin{tabular}{lllll}
\hline Muscles/elasticity(kPa) & Total $(\mathbf{n}=\mathbf{2 1 2})$ & Male $(\mathbf{n}=\mathbf{1 0 0})$ & Female $(\mathbf{n}=\mathbf{1 1 2})$ & $\mathbf{p}$ value* \\
\hline Tibialis anterior & $25.4 \pm 3.2$ & $25.7 \pm 3.4$ & $25.2 \pm 3.0$ & 0.232 \\
Peroneus longus & $15.7 \pm 1.5$ & $15.9 \pm 1.7$ & $15.6 \pm 1.2$ & 0.157 \\
Gastrocnemius medialis & $12.1 \pm 2.1$ & $12.3 \pm 1.9$ & $11.9 \pm 2.2$ & 0.149
\end{tabular}

${ }^{*}$ The independent samples t-tests were performed and a $p$-value $<0.05$ was considered to indicate statistical significance 
Table 2 Mean elasticity values for TA, PL, GA in different age groups ( $n=212$, mean \pm standard deviation)

\begin{tabular}{lllll}
\hline Muscles/elasticity(kPa) & $\mathbf{4} \mathbf{4} \mathbf{y}(\mathbf{n}=\mathbf{1 0 4})$ & $\mathbf{4 5 - 5 9} \mathbf{y}(\mathbf{n}=\mathbf{8 4})$ & $\mathbf{6 0} \mathbf{y}(\mathbf{n}=\mathbf{2 4})$ & $\mathbf{p}$ value* \\
\hline Tibialis anterior & $25.1 \pm 3.3$ & $25.8 \pm 3.2$ & $25.2 \pm 2.9$ & 0.300 \\
Peroneus longus & $15.4 \pm 1.8$ & $15.5 \pm 1.6$ & $15.4 \pm 1.6$ & 0.962 \\
Gastrocnemius medialis & $12.1 \pm 2.3$ & $12.0 \pm 2.0$ & $12.3 \pm 1.8$ & 0.870 \\
\hline
\end{tabular}

${ }^{*}$ The one-way analysis of variance were performed and a $p$-value $<0.05$ was considered to indicate statistical significance

(age $\leq 44$ years); group II, middle age (age $45-59$ years); group III, elderly (age 60-89 years) [31]. All participants provided oral informed consents. All the procedures described in this study were conducted in accordance with the Declaration of Helsinki and International Council for Harmonisation/Good Clinical Practice Guidelines approved by the institutional review board.

\section{Multimodal ultrasound imaging (two-dimensional, color Doppler, 2D-SWE)}

In this study, all imaging procedures (two-dimensional, color Doppler, and 2D-SWE) were performed using an L15-4 high-frequency linear array probe (4-15 MHz) on a Supersonic Imagine ultrasound system (Aixplorer, Supersonic Imagine, Aix-en-Provence, France) by a sonologist with 3 years of work experience, and all sonograms were reviewed by a senior professional post sonologist with 20 years of work experience. The muscles selected for observation were scanned using two-dimensional and color Doppler ultrasound in transverse and longitudinal sections to assess their echo characteristics and blood flow information, followed by shear-wave elastography (SWE) in longitudinal sections. We used SWE standard mode with the tissue Young's modulus values reported in kilopascals (kPA). The stiffness of muscles was displayed inside the frame by color change from blue (soft) to red (hard) depending on the Young's modulus.

Basic information of the nine suspected-ACS patients with swollen limbs are shown in Table 3 (patients were numbered from one to nine in chronological order of admission). Among them, eight patients had swelling in the calves and one patient had swelling in the wrist (Hereinafter referred to as "affected side"). Therefore, we selected the corresponding muscles as the objects of multimodal ultrasound imaging (TA, GA, pronator teres) before treatment. In addition, the same muscles on the healthy sides (Hereinafter referred to as "unaffected side") were scanned as controls.

Another objective of our study was to observe the normal range of elasticity values of the main muscles in each compartment at the resting state. The imaging depth of deep posterior compartment of the leg was a limitation; hence, for the volunteers, we evaluated TA, $\mathrm{PL}$, and GA muscles in the anterior, lateral, superficial posterior compartments by multimodal ultrasound imaging, and observed whether gender and age were independent effect factors.

\section{Protocols of 2D-SWE}

All participants were in the supine position, their limbs laid naturally on the examination bed, and the muscles were in relaxed state. The central location of the target muscle was selected for the measurement, away from the epimysium. The sonologist was asked to scan a given location using minimized transducer pressure. The elastography function was enabled when the grayscale image at the long axis section was clear, a suitable region of interest (ROI) based on the center of the muscle and clear gray-scale image was chosen. The color reflecting the Young's modulus value was evenly filled and stabilized for at least $5 \mathrm{~s}$ before freezing the image for measurement. Subsequently, circles with diameters of $10 \mathrm{~mm}, 8 \mathrm{~mm}$ or $5 \mathrm{~mm}$ were generated at the center of the range depending on the muscle size. Subsequently, the Young's modulus was automatically calculated as the muscle elasticity values. The Young's modulus for each muscle was measured five times, and the three mean values with the lowest coefficient of variation over the circle were used in this study.

\section{Statistical analysis}

The SPSS software (version 20.1, SPSS, IBM) was used for data analysis. Continuous variables are expressed as mean \pm standard deviation. Normality of the distribution of data were verified by using the Shapiro-Wilk test. The independent samples t-test was used to compare the elasticity values of muscles in men and women. The one-way analysis of variance was used to compare the measurement data of different age groups. Elasticity values of the affected and unaffected sides was analyzed by paired samples t-test, and the independent two-sample mean difference $t$-test was used to compare the measurement data of different treatment groups. A $p$-value $<0.05$ was considered statistically significant. 


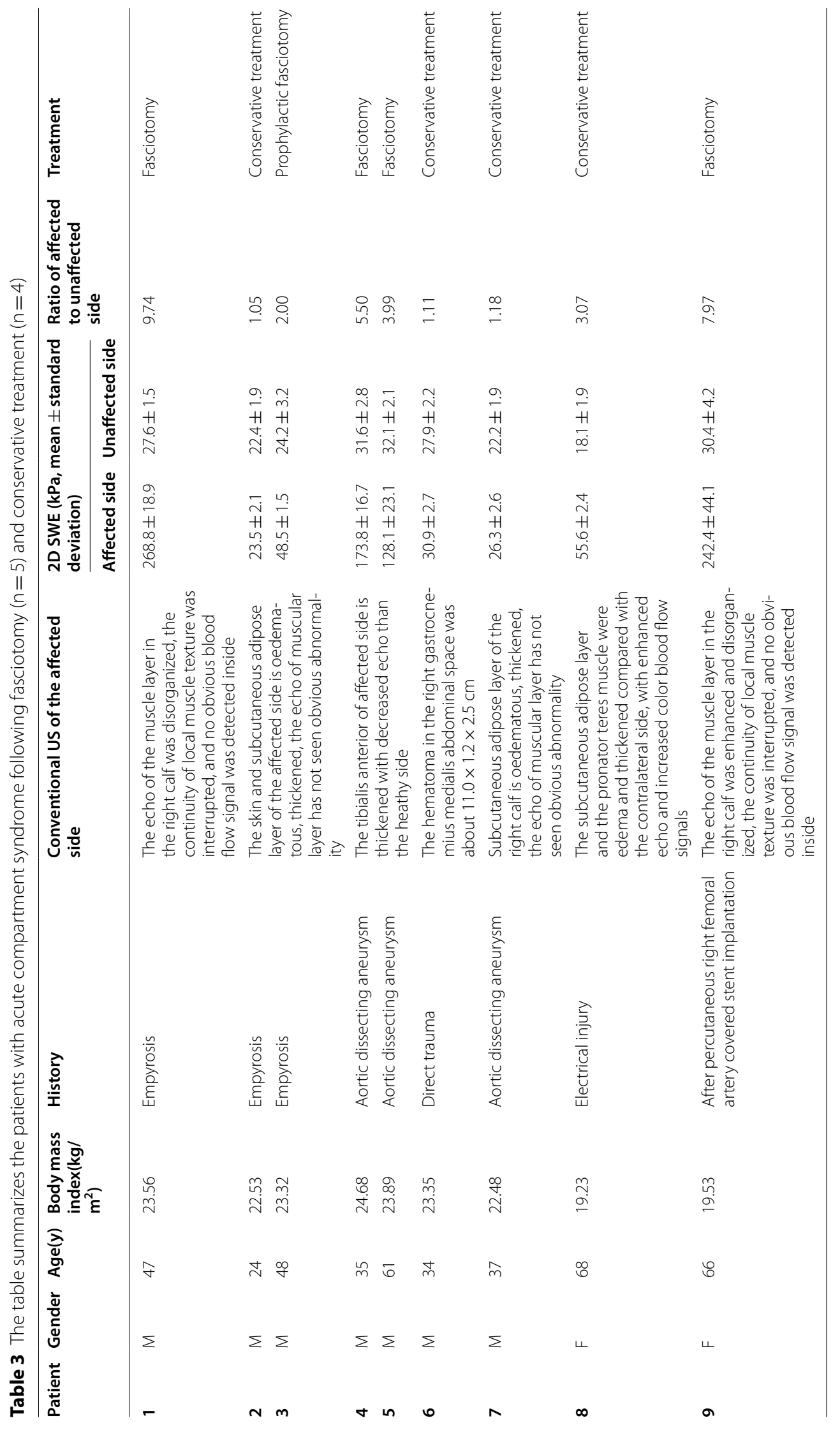




\section{Results}

Among the 212 volunteers, 100 were male participants with an average age of $44.5 \pm 14.8$ years, and 112 were female participants with an average age of $42.6 \pm 12.4$ years. The other nine patients were highly suspected of ACS by clinicians according to their clinical symptoms (such as worsening pain that is out of proportion and increasing analgesic requirements, swelling, paresthesia or anxiety, agitation), their average age was $46.7 \pm 14.7$ years (range: $24-68$ years).

\section{Analysis of differences in muscle elasticity values between genders}

Statistical analysis was conducted on the elasticity values of major muscles (TA, PL, and GA) in each compartment of the calves of 212 volunteers. The results showed that the mean elasticity values of TA, PL, and GA muscles in the relaxed state were $25.4 \pm 3.2 \mathrm{kPa}, 15.7 \pm 1.5 \mathrm{kPa}$, and $12.1 \pm 2.1 \mathrm{kPa}$, respectively. The mean elasticity values of the TA, PL, and GA for men and women were $25.7 \pm 3.4 \mathrm{kPa}, 15.9 \pm 1.7 \mathrm{kPa}, 12.3 \pm 1.9 \mathrm{kPa}$, and $25.2 \pm 3.0 \mathrm{kPa}, 15.6 \pm 1.2 \mathrm{kPa}$, and $11.9 \pm 2.2 \mathrm{kPa}$, respectively ( $p$-values were $0.232,0.157$, and 0.149 , respectively). No statistically significant differences in the elasticity values of the same muscle under the state of relaxation in men and women were observed $(p>0.05$, Table 1; Fig. 1).

\section{Analysis of differences in muscle elasticity values between different age groups}

We divided 212 volunteers into three groups by age, and measured the elasticity values of TA, PL, and GA muscles in each group. Among them, 104 volunteers were $\leq 44$ years old, 84 were 45-59 years old, and 24 were $\geq 60$ years old. The elasticity values of the TA, PL, and GA muscles in different age groups were shown in Table 2 ( $p$-values were $0.300,0.962$, and 0.870 , respectively). The statistical analysis results revealed that there were no statistically significant differences in the elasticity values of the same muscle in the state of relaxation in different age groups $(p>0.05)$.

\section{Analysis of elasticity values of the muscles in the relevant compartments of nine patients with clinical suspicion of ACS}

Among the nine suspected-ACS patients (seven men, two women), three patients were admitted for aortic dissection aneurysm, three patients were admitted for burns of different degrees, one patient was admitted for intramuscular hematoma caused by trauma, one patient was injured by electric shock, and one patient was admitted after percutaneous right femoral artery covered stent implantation.

Conventional ultrasound examination and ultrasound elastography were performed (Fig. 2) for these patients. We found that the patients number $1,4,5$, and 9 not only experienced thickening of the subcutaneous fat layer of the affected limbs, but also experienced thickening of the muscle layer to varying degrees with decreased muscle echo intensity, and the continuity of muscle texture disappeared in some patients. In addition, the elasticity value of the muscle on the affected side was significantly higher than that of the same muscle on the healthy side (the minimum ratio of affected to unaffected side was 3.99). In the other patients (number 2, 3, 6, 7, and $8)$, two-dimensional ultrasound imaging revealed edema and thickening of the subcutaneous adipose layer at corresponding sites, while no obvious abnormalities were found in the echo and thickness of the muscular layer. Furthermore, no significant increase or only a relatively small increase in the elasticity values was measured by 2D-SWE of the muscles on the affected side than on the healthy side (or compared to the healthy volunteers) (Table 3).



Fig. 1 The elasticity values of major muscles (a Tibialis Anterior, $\mathbf{b}$ Peroneus Longus, and $\mathbf{c}$ Gastrocnemius medialis) in each compartment of the calf of a 42 years old volunteer measured by 2D-SWE 

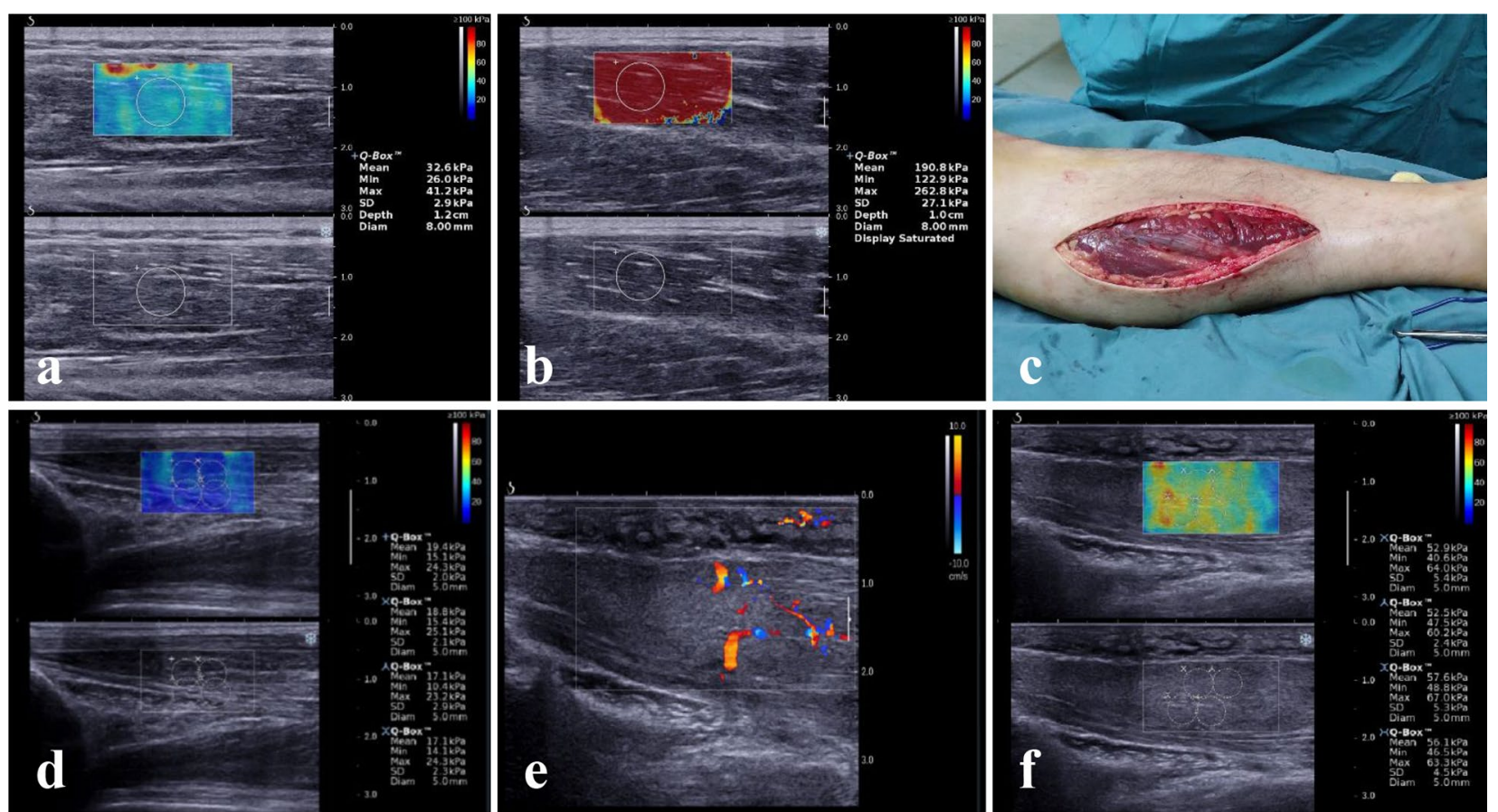

Fig. 2 The images of 2D-SWE and fasciotomy in case 4 of Table $3(a-c)$ : a The elasticity value of TA in the unaffected side. $\mathbf{b}$ The elasticity value of TA in the affected side. $\mathbf{c}$ The patient underwent a fasciotomy for decompression. The images of case 8 of Table 3 (d-e): $\mathbf{d}$ The elasticity values of pronator teres in the unaffected side. e 2D and CDFl images showed that the subcutaneous adipose layer and the pronator teres muscle were edema and thickened with enhanced echo and increased color blood flow signals. $\mathbf{f}$ The elasticity values of pronator teres in the affected side

Finally, five patients (number 1, 3, 4, 5, and 9) were treated with surgical fasciotomy for decompression, and four patients (number 2, 6, 7, and 8) were treated conservatively (non-surgical treatment). In patients number $1,4,5$, and 9 , obvious muscle necrosis was found after surgical fasciotomy. In patient number 3 , the clinician performed a prophylactic incision based on the progression of the disease, and the muscle was found intact during the surgery. All four patients received conservative treatment with satisfactory results, and the limb swelling gradually disappeared and returned to normal.

The elasticity values of the muscles measured by 2D-SWE in patients who underwent different types of treatments are shown in Table 4 . A statistically significant difference in the elasticity values of the muscle on the affected and unaffected sides in the fasciotomy group $(p<0.05, \mathrm{n}=5)$ was observed. In contrast, there was no difference in the elasticity values of the muscle on the affected and unaffected sides in the conservative group $(p>0.05, \mathrm{n}=4)$. There was a statistically significant difference in the elasticity values of the muscle on the affected side in the two groups $(p<0.05)$.
Table 4 Comparison of the elasticity values by using 2D-SWE according to the type of treatment

\begin{tabular}{lcc}
\hline Type of treatment & $\begin{array}{l}\text { 2D-SWE }(\mathbf{k P a}, \\
\text { mean } \pm \text { standard } \\
\text { deviation) }\end{array}$ & p value \\
\hline Fasciotomy $(n=5)$ & $172.3 \pm 72.4$ & $0.019^{(1)}$ \\
$\quad$ Affected side & $29.2 \pm 2.7$ & $0.022^{(2)}$ \\
$\quad$ Unaffected side & \\
Conservative treatment $(n=4)$ & $34.1 \pm 12.7$ & \\
$\quad$ Affected side & $22.7 \pm 3.5$ & \\
$\quad$ Unaffected side & \\
\hline
\end{tabular}

(1) Difference between intergroup (Affected side, Fasciotomy VS conservative treatment), the independent two-sample mean difference $t$-test was performed

(2) Difference between intragroup (Affected side VS unaffected side), the paired sample t-test was perform; A $p$-value $<0.05$ was considered statistically significant

\section{Discussion}

The diagnosis of ACS is challenging, particularly in specific groups, such as children and patients with consciousness disorder who fail to express their chief complaints [32], and to date, the only effective treatment is surgical fasciotomy. Although ACS is generally considered as an "orthopedic emergency," frequent delays exist 
in the time from initial assessment to diagnosis and in the time from diagnosis to surgery in patients with ACS [33]. Delayed fasciotomy is the most important factor contributing to a poor prognosis, with potentially catastrophic consequences, such as permanent sensory impairment, ischemic contracture, muscle dysfunction, amputation, and even death. Hence, early and timely diagnosis and prediction of the incidence of ACS are conducive for prevention and treatment, and protection of the limb function for the patients.

Currently, the diagnosis of ACS is based on physical examination and repeated needle sticks over a short time frame to measure ICP. A method for the accurate and reproducible diagnosis of ACS, especially for the patients with obtunded, polytrauma, or consciousness disturbance, is yet to be developed [34]. The symptoms and signs indicative of ACS have been defined as the 5Ps: pain out of proportion, pallor, paresthesias, paralysis, and pulselessness. Moreover, paralysis, and pulselessness, pallor, and reduced capillary refill time are the late clinical signs. Although previous studies have indicated that combining clinical symptoms and signs raises the sensitivity for diagnosing ACS, and an association of clinical findings with compartment syndrome seems evident, the predictive value of the clinical findings for the diagnosis of compartment syndrome is yet to be delineated [35]. It is not enough to rely solely on the clinical manifestations, especially when patients with impaired consciousness and children are involved [36].

Pressure measurement is the standard method for the diagnosis of ACS, but it is an invasive testing technique, and the process is painful and difficult to repeat. Moreover, most of these invasive methods can only perform "point detection" on the tissue pressure in the osteofascial compartment, which cannot fully reflect the pressure in multiple osteofascial compartments, and it is not convenient for continuous observation of the changes of ICP. The accuracy and reliability of these invasive methods has been questioned $[37,38]$. Therefore, it is not practical in clinical practice, and most clinicians do not adopt this method, but make diagnosis based on clinical experience and $5 \mathrm{P}$ manifestations. There is an urgent clinical need for a less invasive or noninvasive, reliable, safe, and accurate assessment method for the early prevention or treatment of ACS before irreversible muscle ischemia occurs.

Ultrasonography has been widely applied in the diagnosis of musculoskeletal diseases, and some researchers, both at home and abroad, have tried to apply ultrasound imaging in the evaluation of compartment syndrome [11, $39,40]$. Ultrasonic imaging can clearly show the room form and structure of the crus fascia, and when ACS occurs, the two-dimensional images of gray-scale sonography, cross-sectional area of the anterior compartment of the lower leg, pretibial artery diameter, and blood flow dynamics parameters are characteristic changes, and these characteristic changes have good correlation with the fascia indoor pressure and clinical treatment [41]. Gershuni et al. used ultrasound to measure the anterior compartment thickness of the lower leg to evaluate the osteofascial pressure [42], and Mühlbacher et al. investigated the feasibility of noninvasive ultrasound-guided angle measurement as a surrogate of increased pressure in a model of ACS [43]. 2D-SWE is a technique to objectively quantify the elasticity value of the tissues, and has substantial potential for characterizing different soft tissues for clinical diagnosis [12]. It shows high reproducibility when used in the assessment of the liver, breast, and thyroid, however, its application in musculoskeletal diseases still needs further study. 2D-SWE can dynamically quantify and evaluate the real-time changes in muscle elasticity value, and obtain the changes in passive (static) muscle stiffness, dynamic muscle stiffness, and active muscle stiffness under various states by evaluating the viscoelastic characteristics of the muscle [44-46].

ACS could result in an increase in the internal pressure of the compartment and the corresponding increase in muscle stiffness in the compartment. We can indirectly reflect the intra-compartment pressure by measuring muscle stiffness in the compartment through 2D-SWE. Currently, an increasing number of studies are being conducted on the application of 2D-SWE to evaluate skeletal muscle stiffness in different parts of the healthy population, and increasing methodological studies on measurement of skeletal muscle stiffness, which fully reflects the feasibility of 2D-SWE in evaluating muscle stiffness, are being conducted $[11,40]$.

In this study, we measured the elasticity values of major muscles in each calf's compartment of 212 healthy volunteers under the relaxed state by $2 \mathrm{D}-\mathrm{SWE}$, and found that there were no significant differences in the elasticity values of the same muscle in volunteers of different ages and genders under the relaxed state, which provided a basic reference value for our further study. Therefore, we believe that 2D-SWE technology is expected to be a new and effective way to diagnose ACS. In our study, 2D-SWE technique was used to measure the elasticity value of the same muscle on the affected and unaffected sides of the patients who were suspected of ACS, and comparison analysis was conducted. We found that the elasticity value was increased in different degrees on the affected side than on the unaffected side, and the increase of elasticity values were consistent with the patient's clinical manifestations and the tension of corresponding parts. Therefore, we hypothesized that the elasticity values of muscles in compartment measured by 2D-SWE could indirectly reflect the intra-compartment pressure. 
Based on our findings, we suggest that 2D-SWE offers the following advantages over invasive methods such as pressure measurement and subjective assessments based on clinical manifestations: (1) The elasticity value of muscle in compartment was measured by 2D-SWE noninvasively; (2) It can be used to measure the elasticity values of different muscles in different compartments, as well as to measure different parts of the same muscle conveniently; (3) The measurement by 2D-SWE can be repeated and is easy to operate without pain; (4)2D-SWE can also be used for dynamic monitoring and evaluation, providing real-time two-dimensional sonogram of muscles and changes in elasticity value to clinicians, and most importantly the basis evidence for treatment mode selection and adjustment.

This exploratory study has several limitations. First, we assumed that muscle elasticity values at different time periods during the occurrence of ACS could be obtained through massive data analysis, so as to provide clinicians with warning elasticity values that would require surgical fasciotomy. But only nine cases were included in this study, more cases and data need to be collected for further improvement. Second, almost all the patients under observation were critical patients in the intensive care unit, which means that it is a tough road of diverting them to the ultrasound department for examination. Real-time monitoring required us to repeatedly enter the intensive care unit with ultrasonic instruments, which is time-consuming and laborious. We hope that with the continuous development and progress of technology, portable bedside ultrasonic instruments could be equipped with 2D-SWE function, making our operation more convenient. In summary, real-time shear-wave ultrasound elastography has a great potential for the evaluation of the mechanical properties of the skeletal muscles. However, compared with clinical evaluation and other methods, choosing various measurement parameters and standardizing the inspection procedure are still issues that need further assessment.

\section{Conclusions}

Two-dimensional shear-wave ultrasound elastography can not only visually observe muscle damage and related blood flow information in the compartment by using two-dimensional and color Doppler imaging, but can also noninvasively measure the elasticity values of muscles, which can indirectly reflect the intra-compartment pressure. When the ACS occurs, the muscle stiffness of the affected limb increases significantly. 2D-SWE is expected to be a new technique to evaluate ACS which may provide a potential basis for the clinical diagnosis and treatment of ACS and could be used as an auxiliary diagnostic method for ACS. In addition, further studies on muscle SWE standardization in different compartments are needed.

\section{Acknowledgements \\ Thanks for the support of the Cardiac Surgery Intensive Care Unit.}

\section{Authors' contributions}

YM and GH conceived the study. ZJ, ZWF, ZHH, SL, LLN, SYY and GX recruited patients and conducted the study. Data analysis was performed by ZJ.ZJ, YM and GH participated in the writing of the article. All authors read and approved the final manuscript.

\section{Funding}

This research did not receive any specific grant from funding agencies in the public, commercial, or not-for-profit sectors.

\section{Availability of data and materials}

The datasets used and analyzed during the current study are available from the corresponding author on reasonable request.

\section{Declarations}

Ethics approval and consent to participate

The ultrasound examined used in this study is a completely noninvasive method, and all participants were aware and willing to participate in the study, so they provided oral informed consents. All the procedures described in this study were conducted in accordance with the Declaration of Helsinki and International Council for Harmonisation/Good Clinical Practice Guidelines approved by the institutional review board.

\section{Consent for publication}

Not applicable.

\section{Competing interests}

The authors declare that they have no competing interests.

Received: 8 September 2021 Accepted: 30 November 2021

Published online: 15 December 2021

\section{References}

1. Via AG, Oliva F, Spoliti M, Maffulli N. Acute compartment syndrome. Muscles Ligaments Tendons J. 2015;5:18-22.

2. Mauser N, Gissel H, Henderson C, et al. Acute lower-leg compartment syndrome. Orthopedics. 2013;36(8):619-24.

3. Ombregt L. Applied anatomy of the lower leg, ankle and foot. A System of Orthopaedic Medicine: Third Edition. 2013. e287-e298.

4. Hammerberg EM, Whitesides TE, Seiler JG. The reliability of measurement of tissue pressure in compartment syndrome. J Orthop Trauma. 2012;26(1):24-31.

5. Von Keudell AG, Weaver MJ, Appleton PT, Bae DS, Dyer GSM, Heng M, Jupiter JB, Vrahas MS. Diagnosis and treatment of acute extremity compartment syndrome. The Lancet. 2015;386:1299-310.

6. Finkelstein JA, Hunter GA, Hu RW. Lower limb compartment syndrome: course after delayed fasciotomy. J Trauma. 1996:40:342-4.

7. Vaillancourt C, Shrier I, Falk M, Rossignol M, Vernec A, Somogyi D. Quantifying delays in the recognition and management of acute compartment syndrome. CJEM. 2001;3:26-30.

8. Harris IA, Kadir A, Donald G. Continuous compartment pressure monitoring for tibia fractures: does it influence outcome? J Trauma. 2006;60:1330-5.

9. Duckworth AD, McQueen MM. The diagnosis of acute compartment syndrome: a critical analysis review. JBJS Rev. 2017;5:e1.

10. Taljanovic MS, Gimber LH, Becker GW, et al. Shear-wave elastography: basic physics and musculoskeletal applications. Radiographics. 2017;37(3):855-70. 
11. Brandenburg JE, Eby SF, Song P, Zhao H, Brault JS, Chen S, An KN. Ultrasound elastography: the new frontier in direct measurement of muscle stiffness. Arch Phys Med Rehabil. 2014;95:2207-19.

12. Sarvazyan AP, Urban MW, Greenleaf JF. Acoustic waves in medical imaging and diagnostics. Ultrasound Med Biol. 2013;39(7):1133-46.

13. Sarvazyan AP, Rudenko OV, Swanson SD, Fowlkes JB, Emelianov SY. Shear wave elasticity imaging: a new ultrasonic technology of medical diagnostics. Ultrasound Med Biol. 1998;24:1419-35.

14. Badarinza M, Serban O, Maghear L, Bocsa C, Micu M, Porojan MD, Chis BA, Albu A, Fodor D. Multimodal ultrasound investigation (grey scale, Doppler and 2D-SWE) of salivary and lacrimal glands in healthy people and patients with diabetes mellitus and/or obesity, with or without sialosis. Med Ultrason. 2019:21:257-64.

15. Athanasiou A, Tardivon A, Tanter M, Sigal-Zafrani B, Bercoff J, Deffieux T, Gennisson JL, Fink M, Neuenschwander S. Breast lesions: quantitative elastography with supersonic shear imaging-preliminary results. Radiology. 2010;256:297-303

16. Sun Y, Ma C, Liang X, Wang R, Fu Y, Wang S, Cui L, Zhang C. Reproducibility analysis on shear wave elastography (SWE)-based quantitative assessment for skin elasticity. Medicine. 2017:96(19):e6902.

17. Jamialahmadi T, Nematy M, Jangjoo A, Goshayeshi L, Rezvani R, Ghaffarzadegan K, Nooghabi MJ, Shalchian P, Zangui M, Javid Z, Doaei S, Rajabzadeh F. Measurement of liver stiffness with 2D-shear wave elastography (2D-SWE) in bariatric surgery candidates reveals acceptable diagnostic yield compared to liver biopsy. Obes Surg. 2019;29:2585-92.

18. Arda K, Ciledag N, Aktas E, Aribas BK, Köse K. Quantitative assessment of normal soft-tissue elasticity using shear-wave ultrasound elastography. AJR Am J Roentgenol. 2011;197:532-6.

19. Grgurevic I, Puljiz Z, Brnic D, Bokun T, Heinzl R, Lukic A, Luksic B, Kujundzic $M$, Brkljacic B. Liver and spleen stiffness and their ratio assessed by real-time two dimensional-shear wave elastography in patients with liver fibrosis and cirrhosis due to chronic viral hepatitis. Eur Radiol. 2015:25:3214-21.

20. Shinohara M, Sabra K, Gennisson JL, Fink M, Tanter M. Real-time visualization of muscle stiffness distribution with ultrasound shear wave imaging during muscle contraction. Muscle Nerve. 2010;42:438-41.

21. Kuo WH, Jian DW, Wang TG, Wang YC. Neck muscle stiffness quantified by sonoelastography is correlated with body mass index and chronic neck pain symptoms. Ultrasound Med Biol. 2013;39:1356-61.

22. Carpenter EL, Lau HA, Kolodny EH, Adler RS. Skeletal muscle in healthy subjects versus those with gne-related myopathy: evaluation with shearwave US - a pilot study. Radiology. 2015:277:546-54

23. Rosskopf AB, Ehrmann C, Buck FM, Gerber C, Flück M, Pfirrmann CW. Quantitative shear-wave US elastography of the supraspinatus muscle: reliability of the method and relation to tendon integrity and muscle quality. Radiology. 2016;278:465-74.

24. Ruan Z, Zhao B, Qi H, Zhang Y, Zhang F, Wu M, Shao G. Elasticity of healthy Achilles tendon decreases with the increase of age as determined by acoustic radiation force impulse imaging. Int J Clin Exp Med. 2015;8:1043-50.

25. Aubry S, Nueffer JP, Tanter M, Becce F, Vidal C, Michel F. Viscoelasticity in Achilles tendonopathy: quantitative assessment by using real-time shearwave elastography. Radiology. 2015:274:821-9.

26. Wu CH, Chen WS, Wang TG. Elasticity of the coracohumeral ligament in patients with adhesive capsulitis of the shoulder. Radiology. 2016;278:458-64

27. Kantarci F, Ustabasioglu FE, Delil S, Olgun DC, Korkmazer B, Dikici AS, Tutar O, Nalbantoglu M, Uzun N, Mihmanli I. Median nerve stiffness measurement by shear wave elastography: a potential sonographic method in the diagnosis of carpal tunnel syndrome. Eur Radiol. 2014;24:434-40.

28. Andrade RJ, Nordez A, Hug F, Ates F, Coppieters MW, Pezarat-Correia $P$, Freitas SR. Non-invasive assessment of sciatic nerve stiffness during human ankle motion using ultrasound shear wave elastography. J Biomech. 2016:49:326-31.

29. Steinberg BD. Evaluation of limb compartments with increased interstitial pressure. An improved noninvasive method for determining quantitative hardness. J Biomech. 2005;38:1629-35.

30. Shuler MS, Reisman WM, Kinsley TL, Whitesides TE Jr, Hammerberg EM, Davila EM, et al. Correlation between muscle oxygenation and compartment pressure in acute compartment syndrome of the leg. J Bone Joint Surg Am. 2010;92:863-70.
31. Ahmad OB, Boschi-Pinto C, Lopez AD, Murray CJ, Lozano R, Inoue M. Age Standardization of Rates: a new WHO standard [Internet]. Geneva: World Health Organization; 2001.

32. Botanlioglu H, Kantarci F, Kaynak G, Unal Y, Ertan S, Aydingoz O, Erginer R, Unlu MC, Mihmanli I, Babacan M. Shear wave elastography properties of vastus lateralis and vastus medialis obliquus muscles in normal subjects and female patients with patellofemoral pain syndrome. Skeletal Radiol. 2013:42:659-66.

33. Shore BJ, Glotzbecker MP, Zurakowski D, Gelbard E, Hedequist DJ, Matheney TH. Acute compartment syndrome in children and teenagers with tibial shaft fractures: incidence and multivariable risk factors. J Orthop Trauma. 2013;27:616-21.

34. Harvey EJ, Sanders DW, Shuler MS, Lawendy AR, Cole AL, Alqahtani SM, Schmidt AH. What's new in acute compartment syndrome? J Orthop Trauma. 2012;26:699-702

35. Ulmer T. The clinical diagnosis of compartment syndrome of the lower leg: are clinical findings predictive of the disorder? J Orthop Trauma. 2002;16:572-7.

36. Guo J, Yin Y, Jin L, Zhang R, Hou Z, Zhang Y. Acute compartment syndrome: cause, diagnosis, and new viewpoint. Medicine (Baltimore). 2019;98(27):e16260

37. Al-Dadah OQ, Darrah C, Cooper A, Donell ST, Patel AD. Continuous compartment pressure monitoring vs. clinical monitoring in tibial diaphyseal fractures. Injury. 2008;39:1204-9.

38. Prayson MJ, Chen JL, Hampers D, Vogt M, Fenwick J, Meredick R. Baseline compartment pressure measurements in isolated lower extremity fractures without clinical compartment syndrome. J Trauma. 2006;60:1037-40

39. Drakonaki EE, Allen GM, Wilson DJ. Ultrasound elastography for musculoskeletal applications. Br J Radiol. 2012;85:1435-45.

40. Berko NS, Fitzgerald EF, Amaral TD, Payares M, Levin TL. Ultrasound elastography in children: establishing the normal range of muscle elasticity. Pediatr Radiol. 2014:44:158-63.

41. Liu ZH, Liang XR, Shi X, Fu Q, Wu ZJ, Chen JJ. The application of color doppler Ultrasound in acute osteofascial compartment syndrome in legs. Chin J Ultrasound Med. 2009:25:1165-8.

42. Gershuni DH, Gosink BB, Hargens AR, Gould RN, Forsythe JR, Mubarak SJ, Akeson WH. Ultrasound evaluation of the anterior musculofascial compartment of the leg following exercise. Clin Orthop Relat Res. 1982;167:185-90.

43. Mühlbacher J, Pauzenberger R, Asenbaum U, Gauster T, Kapral S, Herkner $H$, Duma A. Feasibility of ultrasound measurement in a human model of acute compartment syndrome. World J Emerg Surg. 2019;14:4.

44. Paluch Ł, Nawrocka-Laskus E, Wieczorek J, Mruk B, Frel M, Walecki J. Use of ultrasound elastography in the assessment of the musculoskeletal system. Pol J Radiol. 2016;81:240-6.

45. Rominger MB, Kälin P, Mastalerz M, Martini K, Klingmüller V, Sanabria S, Frauenfelder $T$. Influencing factors of 2D shear wave elastography of the muscle — an ex vivo animal study. Ultrasound Int Open. 2018;4:E54-60.

46. Chen J, O'Dell M, He W, Du LJ, Li PC, Gao J. Ultrasound shear wave elastography in the assessment of passive biceps brachii muscle stiffness: influences of sex and elbow position. Clin Imaging. 2017;45:26-9.

\section{Publisher's Note}

Springer Nature remains neutral with regard to jurisdictional claims in published maps and institutional affiliations. 